\title{
Phase diagrams of colloidal spheres with a constant zeta-potential
}

\author{
Frank Smallenburg, ${ }^{1}$ Niels Boon, ${ }^{2, a)}$ Maarten Kater, ${ }^{2}$ Marjolein Dijkstra, ${ }^{1}$ and \\ René van Roij ${ }^{2}$ \\ ${ }^{1}$ Soft Condensed Matter, Debye Institute for Nanomaterials Science, Utrecht University, Princetonplein 5 , \\ 3584 CC Utrecht, The Netherlands \\ ${ }^{2}$ Institute for Theoretical Physics, Utrecht University, Leuvenlaan 4, 3584 CE Utrecht, The Netherlands
}

(Received 29 September 2010; accepted 26 January 2011; published online 18 February 2011)

\begin{abstract}
We study suspensions of colloidal spheres with a constant zeta-potential within Poisson-Boltzmann theory, quantifying the discharging of the spheres with increasing colloid density and decreasing salt concentration. We use the calculated renormalized charge of the colloids to determine their pairwise effective screened-Coulomb repulsions. Bulk phase diagrams in the colloid concentration-salt concentration representation follow, for various zeta-potentials, by a mapping onto published fits of phase boundaries of point-Yukawa systems. Although the resulting phase diagrams do feature facecentered cubic and body-centered cubic phases, they are dominated by the (re-entrant) fluid phase due to the colloidal discharging with increasing colloid concentration and decreasing salt concentration. (C) 2011 American Institute of Physics. [doi:10.1063/1.3555627]
\end{abstract}

\section{INTRODUCTION}

Charged colloidal particles suspended in a liquid electrolyte are interesting soft-matter systems that have generated fundamental as well as industrial attention for decades. ${ }^{1}$ Understanding the stability and phase behavior of these systems as a function of colloid concentration and ionic strength is an important theme in many of these studies. A key role is played by the electrostatic repulsions between the colloidal spheres, which are not only capable of stabilizing suspensions against irreversible aggregation due to attractive Van der Waals forces, ${ }^{2}$ but are also the driving force for crystallization, ${ }^{3}$ provided the surface charge on the colloids is high enough and the range of the repulsions long enough. ${ }^{1-3}$ The classic theory that describes the electrostatic repulsions between charged colloidal particles in suspension goes back to the 1940s, when Derjaguin, Landau, Verwey, and Overbeek (DLVO) found, within linear screening theory, that suspended spheres repel each other by screened-Coulomb (Yukawa) interactions. ${ }^{4,5}$ The strength of these repulsions increases with the square of the colloidal charge, and they decay exponentially with particle-particle separation on the length scale of the Debye screening length of the solvent. ${ }^{6}$ This pairwise Yukawa form is a corner stone of colloid science and can explain a large number of observations. ${ }^{1-3}$ For instance, the experimentally observed crystallization of charged colloidal spheres into body-centered cubic (bcc) and face-centered cubic (fcc) phases upon increasing the colloidal packing fraction at low and high salt concentrations, ${ }^{7-10}$ respectively, is in fair agreement with simulations of Yukawa systems. ${ }^{11-14}$ Interestingly, in these simulation studies, as well as in many other studies, ${ }^{15-19}$ the charge of the colloids is assumed to be independent of the colloid density and the salt concentration.

Experiments on deionized aqueous suspensions of highly charged spherical latex colloids with ionizable

\footnotetext{
a) Author to whom correspondence should be addressed. Electronic mail: n.j.h.boon@uu.nl.
}

sulphate groups showed indeed evidence for an effective charge that is independent of volume fraction using elasticity and conductivity measurements. ${ }^{20-22}$ Differences between the effective charge obtained from conductivity and elasticity measurements were attributed to either charge renormalization ${ }^{15}$ or macroion shielding due to many-body effects. ${ }^{23-25}$ Similar conclusions were obtained for silica particles with ionizable carboxylate groups on the surface. ${ }^{26}$

The constant-charge assumption was argued to break down, however, in some recent studies where the electrostatic repulsions were argued to be reduced with increasing colloid concentration. Biesheuvel, ${ }^{27}$ for instance, argues that experimental equilibrium sedimentation-diffusion profiles of charged silica spheres in ethanol at extremely low salt concentration $s^{28}$ are better fitted by a charge-regulation model than by a constant-charge model..$^{29}$ More recent evidence for a concentration-dependent colloidal charge stems from re-entrant melting and re-entrant freezing observations of PMMA spheres in a solvent mixture of cis-decaline and cyclohexyl bromide, i.e., the phase sequence upon increasing the colloid concentration is fluid-crystal-fluid-crystal. ${ }^{30}$ In addition, direct force measurements between a single pair of colloidal PMMA spheres in hexadecane, a pair that is part of a triplet, and a pair that is part of a multiplet have very recently revealed a significant reduction of the force with increasing number of neighboring particles. ${ }^{31}$ Interestingly, in the three experiments of Refs. 28,30, and 31 the solvent is a nonpolar medium.

In fact, the experimental findings of Ref. 31 could well be interpreted and explained in terms of constant-potential boundary conditions on the colloidal surfaces, rather than the more usual constant-charge assumption. The present article addresses the consequence of constant-potential boundary conditions for the packing fraction-salt concentration phase diagram of Yukawa systems by calculating the colloidal charge and the effective screening length for various zetapotentials as a function of salt and colloid concentrations. 
Perhaps surprisingly, such a study has not yet been performed. In the case of high zeta-potential, this requires nonlinear screening theory, and hence the renormalized rather than the bare colloidal charge determines the effective screenedCoulomb repulsions between the colloids. ${ }^{8,15,18,26,32-36}$ For this reason, we use the renormalized charge throughout. We also compare our constant-potential calculations with those of an explicit charge-regulation model, ${ }^{37-42}$ and conclude that their results are qualitatively similar, and even quantitatively if they are considered as a function of the effective screening length.

\section{MODEL AND THEORY}

We consider $N$ colloidal spheres of radius $a$ in a solvent of volume $V$, temperature $T$, dielectric constant $\epsilon$, and Bjerrum length $\lambda_{B}=e^{2} / \epsilon k_{B} T$. Here $e$ is the elementary charge and $k_{B}$ is the Boltzmann constant. The colloidal density is denoted by $n=N / V$ and the packing fraction by $\eta=(4 \pi / 3) n a^{3}$. The suspension is presumed to be in osmotic contact with a 1:1 electrolyte of Debye length $\kappa^{-1}$ and total salt concentration $2 \rho_{s}$. We are interested in suspensions of charged colloids of which the surface (zeta) potential $\psi_{0}$ rather than the charge $Z e$ is fixed. We will show that this constant-potential condition mimics charge-regulation on the colloidal surfaces fairly accurately. The first goal of this article is to calculate $Z$ as a function of $\eta$ for fixed dimensionless combinations $\kappa a, a / \lambda_{B}$, and $\phi_{0} \equiv e \psi_{0} / k_{B} T$. This result will then be used to quantify the effective Yukawa interactions between pairs of colloids, and hence the phase boundaries between fluid, fcc, and bcc crystalline phases.

In the actual suspension of constant-potential colloidal spheres, the charge distribution of each of the $N$ colloids will be distributed heterogeneously over its surface due to the proximity of other colloids in some directions. This leads to a tremendously complex many-body problem that we simplify here by assuming a spherically symmetric environment for each colloid, which is nevertheless expected to describe the average electrostatic properties realistically. Below we will calculate the electrostatic potential $\psi(r)$ at a radial distance $r$ from a charged colloidal sphere at a given zeta-potential $\psi_{0}$, i.e., at a given value $\psi(a)=\psi_{0}$. The colloidal charge $Z e$ then follows from Gauss's law,

$$
\psi^{\prime}(a)=-\frac{Z e}{\epsilon a^{2}}
$$

where a prime denotes a radial derivative.

We first consider a single colloid in the center of a spherical Wigner-Seitz cell of radius $R$, such that the cell volume equals the volume per particle, $(4 \pi / 3) R^{3}=V / N$, which implies $R=a \eta^{-1 / 3}$. The radial coordinate of the cell is called $r$. We write the ionic density profiles for $r \in(a, R)$ as Boltzmann distributions $\rho_{ \pm}(r)=\rho_{s} \exp (\mp \phi(r))$, with $\phi(r)$ $=e \psi(r) / k_{B} T$ the dimensionless electrostatic potential. Together with the Poisson equation $\nabla^{2} \phi=-4 \pi \lambda_{B}\left(\rho_{+}(r)\right.$ $\left.-\rho_{-}(r)\right)$, this gives rise to the radially symmetric PoissonBoltzmann (PB) equation and boundary conditions (BCs)

$$
\phi^{\prime \prime}(r)+\frac{2}{r} \phi^{\prime}(r)=\kappa^{2} \sinh \phi(r), \quad r \in(a, R) ;
$$

$$
\begin{aligned}
& \phi(a)=\phi_{0} ; \\
& \phi^{\prime}(R)=0,
\end{aligned}
$$

where a prime denotes a derivative with respect to $r$. Note that $\mathrm{BC}$ (4) implies charge neutrality of the cell. Once the solution $\phi(r)$ is found for given $\eta, \kappa a$, and $\phi_{0}$, e.g., numerically on a radial grid, the colloidal charge $Z$ follows from Eq. (1), which we rewrite in dimensionless form as

$$
\frac{Z \lambda_{B}}{a}=-a \phi^{\prime}(a)
$$

From the numerical solutions that we will present below, it turns out that $Z$ decreases monotonically from a finite asymptotic low- $\eta$ (large- $R$ ) value $Z_{0}$ to essentially 0 at $\eta \simeq 1$ (or $R \simeq a$ ).

Within linear-screening theory at low packing fraction, where $\sinh \phi \simeq \phi$, the potential distribution can be solved for analytically, yielding $\phi(r)=\phi_{0} a \exp [-\kappa(r-a)] / r$, such that the colloidal charge takes the asymptotic low- $\eta$ and low- $\phi_{0}$ value

$$
\frac{Z_{0} \lambda_{B}}{a}=(1+\kappa a) \phi_{0} .
$$

In the Appendix, we show that the discharging effect with increasing $\eta$, as found from the nonlinear screening theory discussed above, can also be approximated within linear screening theory, yielding

$$
Z(\eta, \kappa a)=\frac{Z_{0}}{1+\eta / \eta^{*}}, \quad \eta^{*}=\frac{(\kappa a)^{2}}{3(1+\kappa a)},
$$

where $\eta^{*}$ is a crossover packing fraction at which the colloidal charge has decayed to half its dilute-limit value $Z_{0}$ given in Eq. (6). For typical numbers of experimental interest, e.g., $a / \lambda_{B}=100$ and $\kappa a=0.25$, we then find $Z_{0}=125 \phi_{0}$ and $\eta^{*}=0.017$. With $\phi_{0} \simeq 1-2$, which corresponds to a surface potential of $25-50 \mathrm{mV}$, we should expect a few hundred charges in the dilute limit and a significant charge reduction for $\eta \gtrsim 10^{-2}$.

The constant-potential boundary condition that we employ here is supposed to mimic charge regulation on the colloidal surface through an association-dissociation equilibrium of chargeable groups on the surface. Here we consider, as a typical example, the reaction $\mathrm{SA} \Leftrightarrow \mathrm{S}^{+}+\mathrm{A}^{-}$where a neutral surface group $\mathrm{SA}$ dissociates into a positively charged surface group $\mathrm{S}^{+}$and a released anion $\mathrm{A}^{-}$. The chemistry of such a reaction can be characterized by a reaction constant $K$ such that $\left[\mathrm{S}^{+}\right]\left[\mathrm{A}^{-}\right] /[\mathrm{SA}]=K$, where the square brackets indicate concentrations in the vicinity of the surface where the reaction takes place. If we now realize that $Z \propto\left[\mathrm{S}^{+}\right]$, we find for the usual case where $\left[\mathrm{S}^{+}\right] \ll[\mathrm{SA}]$ that $Z \propto 1 /\left[\mathrm{A}^{-}\right]$. For the case that the released anion is of the same species as the anion in the reservoir, such that $\left[\mathrm{A}^{-}\right]=\rho_{s} \exp [\phi(a)]$, we thus have

$$
Z=z \exp (-\phi(a))
$$

where the prefactor $z$, which is a measure for the surface chargeability, ${ }^{43}$ accounts for the chemistry, the surface-site areal density, and the total area of the surface between the colloidal particle and the electrolyte solution. Note that Eq. (8) relates the (yet unknown) colloidal charge $Z$ to the 
(yet unknown) zeta-potential $\phi(a)$, for a given $z$. A closed set of equations for charge-regulated colloids is obtained by combining the PB equation (2) with $\mathrm{BC}$ (4) at the boundary of a spherical Wigner-Seitz cell of radius $R$, with $\mathrm{BC}$ (3) replaced by

$$
a \phi^{\prime}(a)=-\frac{\lambda_{B} z}{a} \exp (-\phi(a))
$$

for some given chargeability $z$. The resulting solution $\phi(r)$ gives the zeta-potential $\phi(a)$ as well as the colloidal charge $Z$ using Eq. (8). When comparing the constant-potential model with the ionic association-dissociation model, we will tune the chargeability $z$ such that the low $-\eta$ results for $Z$ coincide for both models.

It is well known that nonlinear screening effects, in particular counterion condensation in the vicinity of a highly charged colloidal surface, reduce the effective colloidal charge that dictates the screened-Coulomb interactions between the colloids. ${ }^{15,18,32-34,44}$ The so-called renormalized colloidal charge, $Z^{*}$, can be calculated from the electrostatic potential $\phi(r)$ as obtained from the nonlinear PB equation by matching the numerically obtained solution at the edge of the cell to the analytically known solution of a suitably linearized problem. By extrapolating the solution of the linearized problem to the colloidal surface at $r=a$, one obtains the effective charge by evaluating the derivative at $r=a$ using Eq. (5). Following Trizac et al., ${ }^{45}$ the renormalized charge $Z^{*}$ can be written as

$$
\begin{aligned}
\frac{Z^{*} \lambda_{B}}{a}= & -\frac{\tanh \phi_{D}}{\bar{\kappa} a}\left(\left(\bar{\kappa}^{2} a R-1\right) \sinh [\bar{\kappa}(R-a)]\right. \\
& +\bar{\kappa}(R-a) \cosh [\bar{\kappa}(R-a)]),
\end{aligned}
$$

where the "Donnan" potential is defined as $\phi_{D} \equiv \phi(R)$, i.e., the numerically found potential at the boundary of the cell, and where the effective inverse screening length is

$$
\bar{\kappa}=\kappa \sqrt{\cosh \phi_{D}} .
$$

Note that $Z^{*}$ and $\bar{\kappa}$ can be calculated for the constant-potential as well as the association-dissociation model in a spherical cell.

\section{EFFECTIVE CHARGE AND SCREENING LENGTH}

For both the constant surface potential (CSP) and the association-dissociation (AD) model discussed above we calculated the bare colloidal charge $Z$, the effective (renormalized) charge $Z^{*}$, and the effective inverse screening length $\bar{\kappa}$ in the geometry of spherical Wigner-Seitz cells. In Fig. 1 we show $Z \lambda_{B} / a$ (full curves) and $Z^{*} \lambda_{B} / a$ (dashed curves) as a function of packing fraction $\eta$, for two screening constants for both the CSP model (black curves) and the AD model (red curves), in (a) for fixed zeta-potential $\phi_{0}=1$ and in (b) for $\phi_{0}=5$. In all cases the chargeability parameter $z$ of the AD model is chosen so as to agree with the CSP model in the lowdensity limit $\eta \rightarrow 0$. For low packing fractions, the red and black curves show agreement for equal $\kappa a$, by construction. At higher $\eta$ the agreement is only qualitative, and the charges predicted by the AD model exceed those of the CSP model, which should not come as a surprise since the former interpo-

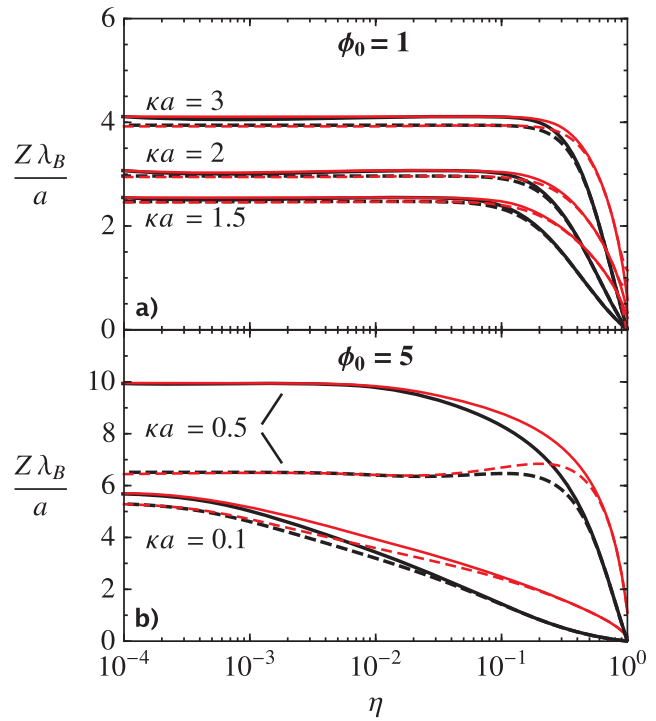

FIG. 1. The bare colloidal charge $Z$ (continuous black curves) and the renormalized charge $Z^{*}$ (dashed black curves), both in units of $a / \lambda_{B}$ (see text), as a function of the colloidal packing fraction $\eta$ for several screening parameters $\kappa a$, for constant surface potentials (a) $\phi_{0}=1$ and (b) $\phi_{0}=5$. The red curves denote $Z$ and $Z^{*}$ as obtained from the association-dissociation model, with the chargeability $z$ chosen such that the surface potential in the dilute limit $\eta \rightarrow 0$ equals $\phi_{0}$.

lates between the constant-charge and the constant-potential model. The close agreement between $Z$ and $Z^{*}$ for all $\kappa a$ at $\phi_{0}=1$ in Fig. 1(a) is also to be expected, since $\phi_{0}=1$ is not far into the nonlinear regime. By contrast, deep in the nonlinear regime of $\phi_{0}=5$, as shown in Fig. 1(b), there is a significant charge renormalization effect such that $Z^{*}<Z$ by a factor of about 1.2 and 1.5 for $\kappa a=0.1$ and $\kappa a=0.5$, respectively. The merging of the red and black curves at high$\eta$ in Fig. 1(b) is due to the reduction of the charge into the linear-screening regime such that $Z=Z^{*}$. The increase of $Z^{*}$ with $\kappa$, as observed in both Figs. 1(a) and 1(b), is in line with well-known charge-renormalization results, ${ }^{15,18,32-34,45}$ and with Eq. (6).

In Figs. 2(a) and 2(b) we plot, for the same zeta-potentials as in Figs. 1(a) and 1(b), the effective screening parameter $\bar{\kappa}$ as a function of $\eta$ for several reservoir screening constants $\kappa$. At low enough $\eta$, where $\kappa R \gg 1$, the two screening constants are indistinguishable from each other in all cases. The reason is that the cell is then large enough for the potential to decay to essentially zero at $r=R$, such that the asymptotic decay of $\phi(r)$ is governed completely by the screening constant $\kappa$ of the background (reservoir) salt concentration. At larger $\eta$, and hence smaller cells, $\phi(R)$ is no longer vanishingly small and the ion concentrations $\rho_{ \pm}(R)$ at $r=R$ deviate considerably from the ionic reservoir concentration $\rho_{s}$. This larger ionic concentration at the cell boundary, which represents an enhanced ion concentration in between the colloidal particles in the true many-body system, leads to a larger effective screening constant $\bar{\kappa}$ with increasing $\eta$ at a fixed $\kappa$, as is shown in Figs. 2(a) and 2(b). Given that larger charges are obtained in the AD model than in the CSP model at high $\eta$, the number of counterions in the cell, and hence $\bar{\kappa}$, is also larger in the AD model. 


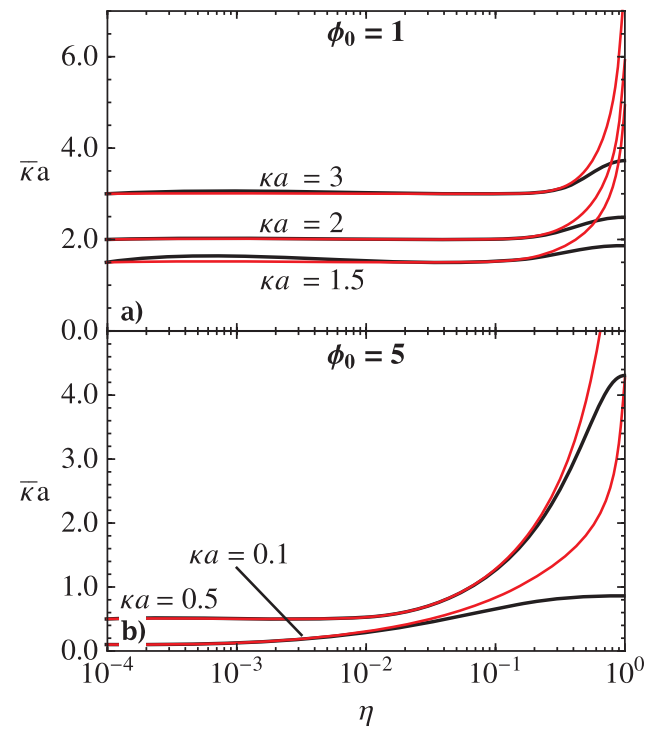

FIG. 2. The effective inverse screening length $\bar{\kappa}$ as a function of the packing fraction $\eta$ for several reservoir screening parameters $\kappa a$, for constant surface potentials (a) $\phi_{0}=1$ and (b) $\phi_{0}=5$ as represented by the black curves. The red curves denote $\bar{\kappa}$ as obtained from the association-dissociation model, with the chargeability $z$ chosen such that the surface potential in the dilute limit $\eta \rightarrow 0$ equals $\phi_{0}$. Note that $\bar{\kappa}=\kappa$ in all cases for $\eta \rightarrow 0$.

\section{EFFECTIVE INTERACTIONS AND PHASE DIAGRAMS}

Once the effective colloidal charge $Z^{*}$ and the effective screening length $\bar{\kappa}^{-1}$ have been determined from the numerical solution of the PB equation in a spherical cell, either for constant-potential or association-dissociation boundary conditions, the effective interactions $u(r)$ between a pair of colloidal particles separated by a distance $r$ follows, assuming DLVO theory, as

$$
\frac{u(r)}{k_{B} T}= \begin{cases}\infty, & r<2 a ; \\ \lambda_{B}\left(\frac{Z^{*} \exp (\bar{\kappa} a)}{1+\bar{\kappa} a}\right)^{2} \frac{\exp (-\bar{\kappa} r)}{r}, & r>2 a,\end{cases}
$$

where we include a short-range hard-core repulsion for overlapping colloids and ignore Van der Waals forces (which is justified for index-matched particles). Note that the pair potential $u(r)$ depends on density-dependent parameters $Z^{*}$ and $\bar{\kappa}$, and, therefore, contains two many-body effects, (i) charge renormalization and (ii) colloidal discharging with increasing density. However, one could expect macroion shielding as another many-body effect. ${ }^{8,26,35,46}$ In states where the pair interaction $u(r)$ is so weak that a fluid phase results, we expect the macroion shielding to be weak; in crystalline states with strong effective pair interactions macroion shielding could also be significant. We expect, however, that the strongest underlying assumption in crystalline states is the spherical cell employed in our calculations. The actual Wigner-Seitz cell in face-centered-cubic and body-centered-cubic crystal phases will probably generate an anisotropic charge distribution on constant-potential colloids and hence anisotropic pair interactions. Such a problem could in principle be tackled with the numerical technique developed in Ref. 47, but due to its numerical involvement such a study is left for future work. Be- low, we will find a surprisingly large parameter regime, where a fluid phase is predicted, which gives an a posteriori justification for the use of the relatively simple pair potential $u(r)$ of Eq. (12).

One could use this pair interaction to simulate (or otherwise calculate) properties of the suspension in a given state point, e.g., whether the system is in a fluid or crystalline state. We restrict our attention here to the limiting case in which the colloidal particles are sufficiently highly charged and/or sufficiently weakly screened, that the pair potential at contact satisfies $u(2 a) \gg k_{B} T$, thereby effectively preventing direct particle-particle contact. In this limit, the suspension can be effectively regarded as a point-Yukawa system that can be completely characterized by only two dimensionless parameters $U$ and $\lambda$ for the strength and the range of the interactions, respectively. They are defined as

$$
\begin{aligned}
& U=\left(\frac{Z^{*} \exp (\bar{\kappa} a)}{1+\bar{\kappa} a}\right)^{2} \frac{\lambda_{B}}{a}\left(\frac{3 \eta}{4 \pi}\right)^{1 / 3}, \\
& \lambda=\bar{\kappa} a\left(\frac{3 \eta}{4 \pi}\right)^{-1 / 3},
\end{aligned}
$$

such that the point-Yukawa interaction potential of interest, in units of $k_{B} T$, reads $U \exp (-\lambda x) / x$ with $x=r(N / V)^{1 / 3}$ the particle separation in units of the typical particle spacing. Note that three dimensionless parameters would have been needed if hard-core contact was not a low Boltzmann-weight configuration, e.g., then the contact-potential $\beta u(2 a)$ (i.e., the inverse temperature), the dimensionless screening parameter $\kappa a$, and the packing fraction $\eta$ would be a natural choice. The mapping of the phase diagram of the point-Yukawa system onto hard-core Yukawa systems has been tested and verified explicitly by computer simulation. ${ }^{14}$

The point-Yukawa system has been studied by simulation in great detail over the years, ${ }^{11-14}$ and by now it is well known this model features a disordered fluid phase and two crystalline phases [(fcc) and (bcc)]. Their first-order phase boundaries are well documented and can accurately be described by curves in the two-dimensional $(\lambda, U)$ plane. Here, we employ the fits for the phase boundaries of point-Yukawa particles that were presented in Ref. 14, which were based on the results of Hamaguchi et al. ${ }^{13}$ The melting-freezing line between the bcc crystal and the fluid is accurately fitted by

$$
\begin{aligned}
\ln U= & 4.670-0.04171 \lambda+0.1329 \lambda^{2}-0.01043 \lambda^{3} \\
& +4.343 \cdot 10^{-4} \lambda^{4}-6.924 \cdot 10^{-6} \lambda^{5}, \\
& \text { for } 0 \leq \lambda \leq 12,
\end{aligned}
$$

and the bec-fcc transition by

$$
\begin{aligned}
\ln U= & 97.65106-150.469699 \lambda+106.626405 \lambda^{2} \\
& -41.67136 \lambda^{3}+9.639931 \lambda^{4}-1.3150249 \lambda^{5} \\
& +0.09784811 \lambda^{6}-0.00306396 \lambda^{7}, \\
& \text { for } 1.85 \leq \lambda \leq 6.8 .
\end{aligned}
$$

Here we exploit these empirical relations as follows. For given dimensionless colloid radius $a / \lambda_{B}$, screening constant $\kappa a$, and various $\eta$, we calculate $Z^{*}$ and $\bar{\kappa} a$ for the CSP and 

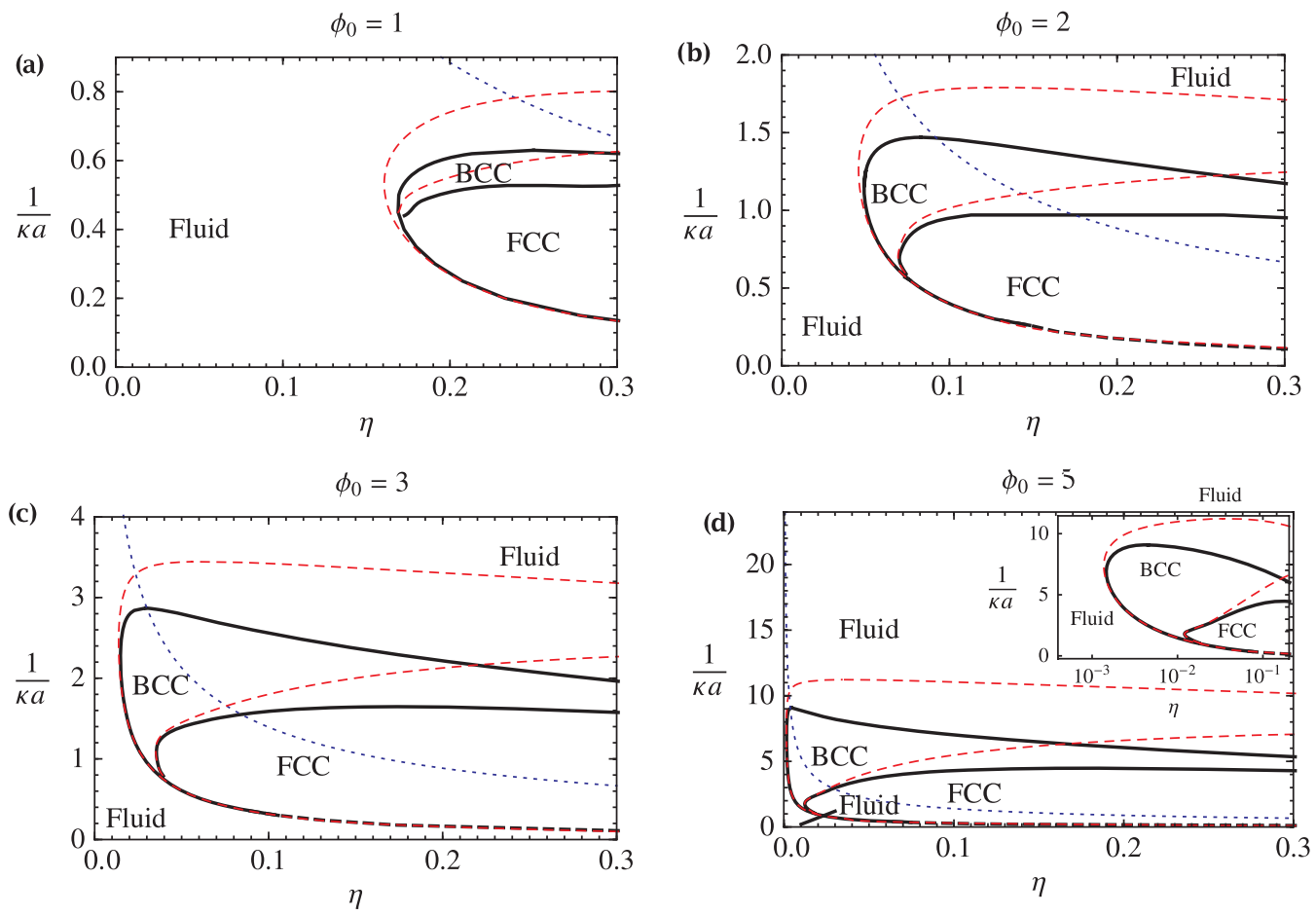

FIG. 3. Phase diagrams in the packing fraction-screening length $\left(\eta, \kappa^{-1}\right)$ representation for constant-potential colloids (radius $\left.a / \lambda_{B}=100\right)$ interacting with the hard-core Yukawa potential of Eq. (12), for surface potentials $\phi_{0}=1,2,3$, and 5. The black lines represent phase boundaries for the constant-potential model, and the red dashed lines for the association-dissociation model with the surface potential equal to $\phi_{0}$ in the dilute limit. The dashed black lines indicate extrapolation of Eq. (15) beyond its strict regime of accuracy. The inset in the phase diagram for $\phi_{0}=5$ represents $\eta$ on a logarithmic scale for clarity. The labels "Fluid," "BCC," and "FCC" denote the stable fluid, bcc, and fcc regions. We note that the very narrow fluid-fcc, fluid-bcc, and fcc-bcc coexistence regions are just represented by single curves. The dotted blue curves represent the estimated crossover-packing fraction $\eta^{*}$ of Eq. (7), beyond which $Z(\eta)<Z(0) / 2$.

the AD model in the spherical cell, as described in Sec. II. These quantities can be used to compute the dimensionless Yukawa parameters $U$ and $\lambda$ from Eqs. (13) and (14), such that their phase and phase boundaries are known from Eqs. (15) and (16).

For $a / \lambda_{B}=100$, Fig. 3 shows the phase diagrams that result from this point-Yukawa mapping procedure in the $\left(\eta,(\kappa a)^{-1}\right)$ representation, for the CSP model (black curves) with surface potentials (a) $\phi_{0}=1$, (b) $\phi_{0}=2$, (c) $\phi_{0}$ $=3$, (d) $\phi_{0}=5$, and for the corresponding AD model (red curves). The dashed lines represent the phase boundary fits of Eqs. (15) and (16) outside their strict $\lambda$-regime of applicability. We restrict attention to $\eta<0.3$, as the point-Yukawa limit breaks down due to strong excluded-volume effects at higher packing fractions. An expected feature is the shift of the freezing curves to lower $\eta$ for higher $\phi_{0}$, due to the higher (renormalized) charge and the stronger repulsions at higher $\phi_{0}$. Due to the higher charges in the AD model, its crystallization regimes (red curves) extend to somewhat lower $\eta$ 's and longer screening lengths than those of the CSP model (black curves). However, the most striking feature of all these phase diagrams is the huge extension of the fluid regime: at high and at low screening length there is no crystalline phase at all (for $\eta$ $<0.3$ ), while at some intermediate salt concentrations the crystal phases are sandwiched in between an ordinary low-density fluid and a re-entrant fluid phase. This re-entrant fluid regime becomes more prominent with increasing zeta-potential $\phi_{0}$. The underlying physics of this
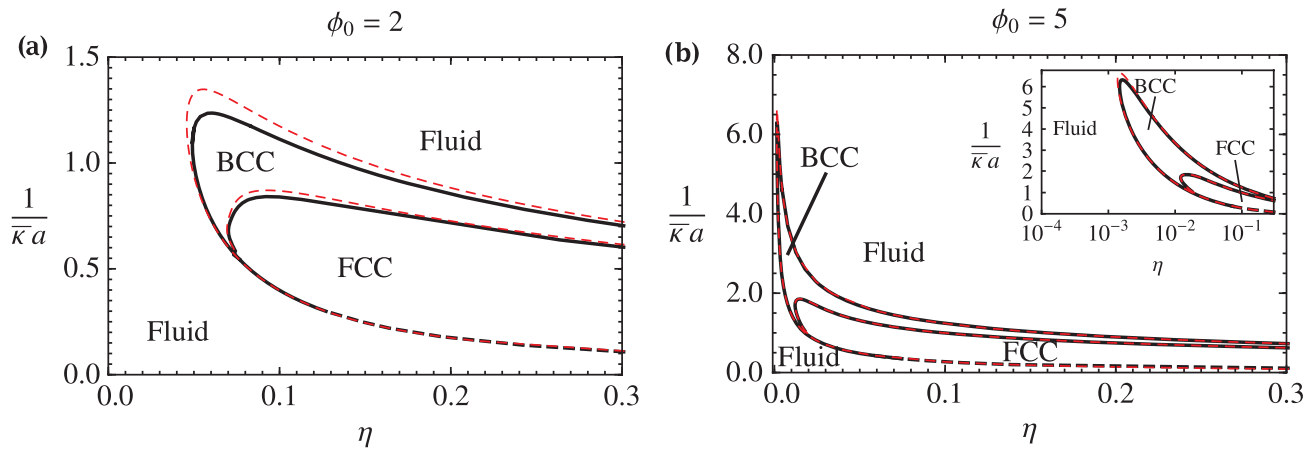

FIG. 4. Phase diagrams in the packing fraction-effective screening length representation $\left(\eta,(\bar{\kappa} a)^{-1}\right)$, for $a / \lambda_{B}=100$, for constant-potential colloids with (a) $\phi_{0}=2$ and (b) $\phi_{0}=5$, as well as for charge-regulated colloids. Lines, symbols, and colors as in Fig. 3 . 


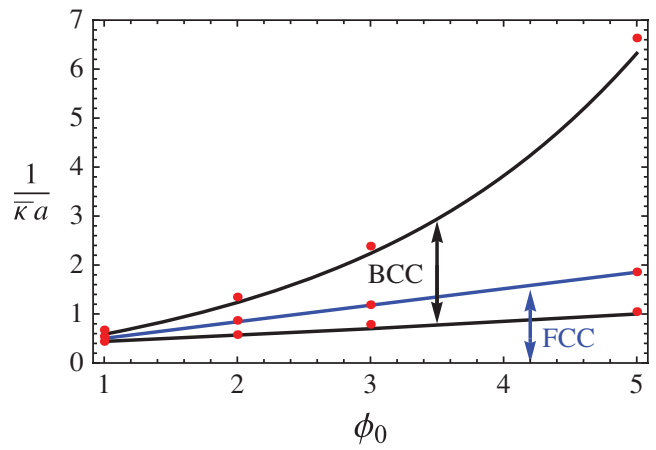

FIG. 5. Maximum and minimum effective screening lengths where bcc and fcc can be found as a function of the surface potential, assuming a constant surface potential for $a / \lambda_{B}=100$. The bcc regime is in between the two black lines, and the fcc regime below the blue line. The red points indicate the results from the AD model.

finite-salt and finite- $\eta$ regime where bcc and fcc crystals exist is the discharging of the colloids with increasing $\eta$ and decreasing salt concentration: (i) although at high salt (small screening length) the colloidal charge is high, the screened-Coulomb interaction is then so short ranged that the system resembles a hard-sphere system that will only crystallize at $\eta \simeq 0.5$; (ii) at low salt (long screening length) the colloidal charge is too low to have seizable repulsions that drive crystallization. Only at intermediate salt and intermediate colloidal packing the charge is high enough and the screening sufficiently long ranged to drive crystallization. The dotted blue curves in Fig. 3 represent the crossover packing fraction $\eta^{*}$ of Eq. (7) beyond which the colloidal charge has been reduced to less than $50 \%$ of its dilute-limit value. Our expression for $\eta^{*}$ indeed roughly coincides with the onset of the re-entrant fluid regime. Equation (7) thus provides a quick guide to estimate where or whether re-entrant melting is to be expected at all. Interestingly, at $\phi_{0}=3$, there are values for $\kappa a$ (albeit in a very narrow range) where a phase sequence fluid-bcc-fcc-bcc is predicted here upon increasing the colloidal packing fraction, showing a re-entrant bcc phase appearing after the fcc crystal. Moreover, for $\eta>0.5$, one expects hard-sphere freezing into a fcc (or hcp) stacking on the basis of hard-sphere interactions, so the fcc phase is then also re-entrant. Clearly, the re-entrant bcc-fcc-bcc transition only occurs in a tiny parameter regime and may well disappear (or appear much more strongly) in more detailed calculations that take, for instance, the nonspherical symmetry of the fcc and bcc phases into account - this would render the colloidal charge distribution heterogeneous and would complicate the calculations considerably. We expect, however, that our main finding-a phase diagram with a huge fluid part and a strongly reduced crystalline part due to the discharging of the colloids with lowering the salt concentration and increasing $\eta$-is a robust prediction.

Experimentally, it is not always possible or convenient to characterize the screening in terms of the Debye length $\kappa^{-1}$ of the (hypothetical) reservoir with which the suspension would be in osmotic equilibrium. For instance, in conductivity measurements at finite colloid concentration, one essentially measures the ionic strength of the sample, which is directly related to the effective screening constant $\bar{\kappa}$ rather than $\kappa$. Also, any measurement of effective colloidal interactions will yield $\bar{\kappa}$. Because of this accessibility of $\bar{\kappa}$, we replot in Fig. 4 the phase diagrams for $\phi_{0}=2$ and $\phi_{0}=5$ of Fig. 3, but now in the $\left(\eta,(\bar{\kappa} a)^{-1}\right)$ representation. While the phase behavior in, for example, a sedimentation experiment will be easier to compare to the phase diagrams shown in Fig. 3, the representation of Fig. 4 could be useful in cases where no ion reservoir is present, while the effective screening length is known. Interestingly, the CSP and AD model are much closer together in Fig. 4 compared to Fig. 3, and the re-entrant fluid phase appears even more pronounced in this representation.

In order to quantify in which finite salt-concentration regime bcc and fcc crystals are expected in a colloidal concentration series $0<\eta<0.3$, we analyze the maximum and minimum values of $\bar{\kappa} a$ at which these two crystal phases can exist, as a function of the zeta-potential $\phi_{0}$, for $a / \lambda_{B}=100$. Fig. 5 shows the resulting screening-length regimes, both for bcc (black curves) and fcc (blue curve), where the lowest screening length for fcc crystals is set to zero because of the hard-sphere freezing into fcc at $\eta=0.5$ even for $1 / \kappa a \rightarrow 0$ - of course we only restricted attention to $\eta<0.3$ until now so strictly speaking also the fcc phase should have had a nonvanishing lower bound. Nevertheless, despite this small inconsistency, Fig. 5 clearly shows not only that a larger zeta-potential gives rise to a larger crystal regime, but also that for all $\phi_{0}$ there is a limiting screening length beyond
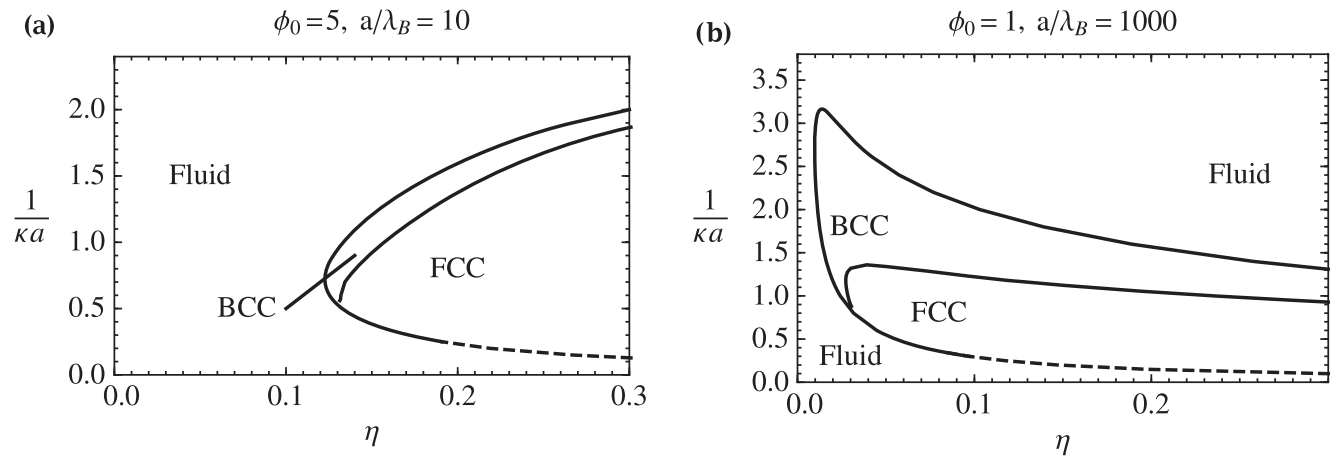

FIG. 6. Phase diagrams in the packing fraction-screening length representation $\left(\eta,(\bar{\kappa} a)^{-1}\right)$, for constant-potential colloids with (a) $\phi_{0}=5$ for $a / \lambda_{B}=10$ and (b) $\phi_{0}=1$ and for $a / \lambda_{B}=1000$. Lines and symbols as in Fig. 3. 
which neither fcc nor bcc crystals can exist, both for the CSP and the AD model.

So far we focussed on $a / \lambda_{B}=100$, which in aqueous suspensions corresponds to a colloidal radius of about $70 \mathrm{~nm}$. However, the colloidal size regime can easily be a factor 10 larger or smaller, and for that reason we also consider the CSP model for $a / \lambda_{B}=10$ and 1000. In Fig. 6, we show the phase diagrams for the smaller colloids with $\phi_{0}=5$ in (a), and for larger colloids with $\phi_{0}=1$ in (b). When compared to the larger colloids $\left(a / \lambda_{B}=100\right)$, the phase diagram for the smaller colloids comes closest to the one shown in Fig. 3(b) for $\phi_{0}=2$, but the reentrant fluid has disappeared completely. Additionally, the smaller particles need a much higher potential to crystallize-the phase diagram for $a / \lambda_{B}=10$ at $\phi_{0}=1$ does not show a crystal phase at all for $\eta<0.3$. Similarly, the larger colloids require a much lower surface potential to resemble the phase diagram shown in Fig. 3(c). Additionally, the range of screening lengths where a reentrant fluid exists is much larger than for $a / \lambda_{B}=100$. Given that $\phi_{0}=5$ is a rather high potential that may be difficult to achieve in reality while $\phi_{0}=1$ is frequently occurring, one concludes that re-entrant melting occurs in the largest salt-concentration regime and is hence easiest observable by tuning the salt, for larger colloids.

\section{SUMMARY AND CONCLUSIONS}

Within a spherical cell model, we have calculated the bare charge $Z$, the renormalized charge $Z^{*}$, and the effective screening length $\bar{\kappa}^{-1}$ of colloidal spheres at a constant zeta-potential $\phi_{0}$. We find from numerical solutions of the nonlinear Poisson-Boltzmann equation that these constant-potential colloids discharge with increasing packing fraction and ionic screening length, in fair agreement with analytical estimates for the dilute-limit charge $Z_{0}$ in Eq. (6) and the typical crossover packing fraction $\eta^{*}$ given in Eq. (7). We also show that the constant-potential assumption is a reasonably accurate description of charge regulation by an ionic association-dissociation equilibrium on the colloidal surface. We use our nonlinear calculations of $Z^{*}$ and $\bar{\kappa}$ to determine the effective screened-Coulomb interactions between the colloids at a given state point, and we calculate the phase diagram for various zeta-potentials by a mapping onto empirical fits of simulated phase diagrams of point-Yukawa fluids. This reveals a very limited regime of bcc and fcc crystals: in order to form crystals, the charge is only high enough and the repulsions only long ranged enough in a finite intermediate regime of packing fraction and salt concentrations; at high $\eta$ or low salt the spheres discharge too much, and at high salt the repulsions are too short ranged to stabilize crystals. In the salt-regime where crystals can exist, the discharging mechanism gives rise to re-entrant phase behaviour, with phase sequences, fluid-bcc-fluid and even fluid-bcc-fcc-bcc (although in an extremely small regime) upon increasing the colloid concentration from extremely dilute to $\eta=0.3$.

The phase behavior of constant-potential or chargeregulated colloids as reported here is quite different from that of constant-charge colloids, for which the pairwise repulsions do not weaken with increasing volume fraction or decreasing salt concentration. As a consequence constant-charge colloids have a much larger parameter-regime where crystals exist and do not show the re-entrant behavior. ${ }^{11-14}$ The most direct comparison is to be made with the constant-charge phase diagrams of Figs. 2 and 4 of Ref. 14, where the charge is fixed such that the surface potential at infinite dilution corresponds to $\phi_{0} \simeq 1$ and 2, respectively. Our theoretical findings can thus be used to gain insight into the colloidal charging mechanism by studying colloidal crystallization regimes as a function of packing fraction and salt concentration.

\section{ACKNOWLEDGMENTS}

Financial support of an NWO-ECHO and an NWO-VICI grant is acknowledged.

\section{APPENDIX: JELLIUM MODEL}

Although it is numerically straightforward to solve the nonlinear PB equation (2) with BCs (3) and (4) in a spherical Wigner-Seitz cell of radius $R$, it may also be convenient to have analytic results that allow for quick estimates of the (order of) magnitude of the colloidal charge $Z$. A standard approach is to linearize the $\sinh \phi(r)$ term of Eq. (2), e.g., with $\phi(r)-\phi(R)$ as the small expansion parameter. The resulting solution is then of the form $\phi(r)=A \exp (-\bar{\kappa} r) / r$ $+B \exp (\bar{\kappa} r) / r+C$, with $\bar{\kappa}$ defined in Eq. (11), $C=\phi(R)$ - $\tanh \phi(R)$, and with integration constants $A$ and $B$ fixed by the two BCs. The algebra involved is, however, not very transparent.

A considerable simplification is achieved if we consider the so-called Jellium model, in which the central colloidal sphere is no longer considered to be surrounded by only cations and anions in a finite cell, but instead by cations, anions and other colloids with charge $Z$ (to be determined). ${ }^{32-34}$ $\mathrm{A}$ nonlinear $\mathrm{PB}$ equation and $\mathrm{BCs}$ can then be written, for $r \geq a$,

$$
\begin{aligned}
& \phi^{\prime \prime}(r)+\frac{2}{r} \phi^{\prime}(r)=\kappa^{2} \sinh \phi(r)-4 \pi \lambda_{B} Z n ; \\
& \phi(a)=\phi_{0} ; \\
& \phi^{\prime}(\infty)=0,
\end{aligned}
$$

where it is assumed that the "other" colloids are distributed homogeneously with density $n$. From this, one derives directly that the asymptotic potential is given by

$$
\sinh \phi(\infty)=\frac{4 \pi \lambda_{B} Z n}{\kappa^{2}}=\frac{3 \eta\left(Z \lambda_{B} / a\right)}{(\kappa a)^{2}} .
$$

Now linearizing $\sinh \phi(r)$ with $\phi(r)-\phi(\infty)$ as the small expansion parameter gives rise to the solution,

$$
\phi(r)=\phi(\infty)+\left(\phi_{0}-\phi(\infty)\right) \frac{\exp (-\tilde{\kappa}(r-a))}{r / a},
$$

where the effective screening length $\tilde{\kappa}^{-1}$ is defined by

$$
\tilde{\kappa}=\kappa \sqrt{\cosh \phi(\infty)} .
$$

We note that the average ion concentrations in the system, within the present linearization scheme, is given by 
$c_{ \pm}=\rho_{s} \exp (\mp \phi(\infty))$, such that the corresponding screening length $\tilde{\kappa}^{-1}$ is given by $\tilde{\kappa}^{2}=4 \pi \lambda_{B}\left(c_{+}+c_{-}\right)$. In other words, the effective screening length $\tilde{\kappa}$ and the asymptotic potential $\phi(\infty)$ of this jellium model play exactly the same role as $\bar{\kappa}$ and $\phi(R)$ that we introduced before in the spherical cell. In particular, $\bar{\kappa}^{-1}$ and $\tilde{\kappa}^{-1}$ can be seen as the actual screening length in the suspension (in contrast to the screening length $\kappa^{-1}$ of the ion reservoir).

From Eq. (A5) the colloidal charge $Z$ follows, using Eq. (5), as the solution of the transcendental equation

$$
\frac{Z \lambda_{B}}{a}=\left(\phi_{0}-\phi(\infty)\right)(1+\bar{\kappa} a)
$$

where one should realize that both $\phi(\infty)$ and $\tilde{\kappa}$ depend on $Z \lambda_{B} / a$ through Eqs. (A4) and (A6). It is possible to solve Eq. (A7) explicitly in the dilute limit. For $\eta=0$ one finds $\phi(\infty)=0$ from Eq. (A4), and hence $Z=Z_{0}$ given by Eq. (6). For finite but low-enough $\eta$ for which $\phi(\infty) \ll 1$, one can ignore $\mathcal{O}\left(\eta^{2}\right)$ contributions, such that $\sinh \phi(\infty) \simeq \phi(\infty)$ and $\cosh \phi(\infty) \simeq 1$, to find Eq. (7) from the self-consistency condition Eq. (A7).

${ }^{1}$ R. J. Hunter, Foundations of Colloid Science 2nd ed. (Oxford University Press, New York, 2001).

${ }^{2}$ J. Israelachvili, Intermolecular and Surface Forces 2nd ed. (Academic, New York, 1991).

${ }^{3} \mathrm{~J}$. L. Barrat and J. P. Hansen, Basic concepts for simple and complex fluids (Cambridge University Press, 2003).

${ }^{4}$ B. Derjaguin and L. Landau, Acta Physicochim. URSS 14, 633 (1941).

${ }^{5}$ E. J. W. Verwey and J. T. G. Overbeek, Theory of Stability of Lyophobic Colloids (Elsevier, Amsterdam, 1948).

${ }^{6}$ J. C. Crocker and D. G. Grier, Phys. Rev. Lett. 73, 352 (1994).

${ }^{7}$ E. B. Sirota, H. D. Ou-Yang, S. K. Sinha, P. M. Chaikin, J. D. Axe, and Y. Fujii, Phys. Rev. Lett. 62, 1524 (1989).

${ }^{8}$ Y. Monovoukas and A. P. Gast, J. Colloid Interface. Sci. 128, 533 (1989).

${ }^{9}$ T. Okubo, Chem. Soc. Faraday Trans. 86, 2871 (1990).

${ }^{10}$ T. Okubo, J. Chem. Phys. 95, 3690 (1991).

${ }^{11}$ M. O. Robbins, K. Kremer, and G. S. Grest, J. Chem. Phys. 88, 3286 (1988).

${ }^{12}$ E. J. Meijer and D. Frenkel, J. Chem. Phys. 94, 2269 (1991).

${ }^{13}$ S. Hamaguchi, R. T. Farouki, and D. H. E. Dubin, Phys. Rev. E 56, 4671 (1997).

${ }^{14}$ A.-P. Hynninen and M. Dijkstra, Phys. Rev. E. 68, 021407 (2003).

${ }^{15}$ S. Alexander, P. M. Chaikin, G. J. Morales, P. Pincus, and D. Hone, J. Chem. Phys. 80, 5776 (1984).
${ }^{16}$ M. E. Leunissen, C. G. Christova, A.-P. Hynninen, C. P. Royall, A. I. Campbell, A. Imhof, M. Dijkstra, R. van Roij, and A. van Blaaderen, Nature (London) 437, 235 (2005).

${ }^{17}$ J. Dobnikar, Y. Chen, R. Rzehak, and H. H. von Grünberg, J. Chem. Phys. 119, 4971 (2003).

${ }^{18}$ B. Zoetekouw and R. Roij, Phys. Rev. Lett. 97, 258302 (2006).

${ }^{19}$ Y. Levin, Rep. Prog. Phys. 65, 1577 (2002).

${ }^{20}$ P. Wette, H. J. Schöpe, and T. Palberg, Colloids Surf., A 222, 311 (2003).

${ }^{21}$ P. Wette, H. J. Schöpe, and T. Palberg, J. Chem. Phys. 116, 10981 (2002).

${ }^{22}$ D. Hessinger, M. Evers, and T. Palberg, Phys. Rev. E 61, 5493 (2000).

${ }^{23}$ R. Klein, H. H. von Grünberg, C. Bechinger, and V. Lobaskin, J. Phys.: Condens. Matter 14, 7631 (2002).

${ }^{24}$ M. Brunner, C. Bechinger, W. Strepp, V. Lobaskin, and H. H. von Grünberg, Europhys. Lett. 58, 926 (2002).

${ }^{25}$ C. Russ, H. H. von Grünberg, M. Dijkstra, and R. van Roij, Phys. Rev. E 66, 011402 (2002).

${ }^{26}$ P. Wette, I. Klassen, D. Holland-Moritz, D. M. Herlach, H. J. Schöpe, N. Lorenz, H. Reiber, T. Palberg, and S. V. Roth, J. Chem. Phys 132, 131102 (2010).

${ }^{27}$ P. M. Biesheuvel, J. Phys.: Condens. Matter 16, L499 (2004).

${ }^{28}$ M. Rasa, B. Erné, B. Zoetekouw, R. van Roij, and A. P. Philipse, J. Phys.: Condens. Matter 17, 2293 (2005).

${ }^{29}$ R. van Roij, J. Phys.: Condens. Matter 15, S3569 (2003).

${ }^{30}$ C. P. Royal, M. E. Leunissen, A.-P. Hynninen, M. Dijkstra, and A. van Blaaderen, J. Chem. Phys. 124, 244706 (2006).

${ }^{31}$ J. W. Merrill, S. K. Sainis, and E. R. Dufresne, Phys. Rev. Lett. 103, 138301 (2009).

${ }^{32}$ E. Trizac, L. Boucqet, and M. Aubouy, Phys. Rev. Lett. 89, 248301 (2002).

${ }^{33}$ E. Trizac and Y. Levin, Phys. Rev. E 69, 031403 (2004)

${ }^{34}$ T. E. Colla, Y. Levin, and E. Trizac, J. Chem. Phys. 131, 074115 (2009).

${ }^{35}$ A. Toyotama, T. Sawada, J. Yamanaka, and K. Kitamura, Langmuir 19, 3236 (2003).

${ }^{36}$ L. F. Rojas-Ochoa, R. Castañeda-Priego, V. Lobaskin, A. Stradner, F. Scheffold, and P. Schurtenberger, Phys. Rev. Lett. 100, 178304 (2008).

${ }^{37}$ B. W. Ninham and V. A. Parsegian, J. Theor. Biol. 31, 405 (1971).

${ }^{38}$ D. Y. C. Chan and D. J. Mitchel, J. Colloid Interface Sci. 227, 152 (2000).

${ }^{39}$ S. H. Behrens and J. Borkovec, J. Chem. Phys. 111, 382 (1999).

${ }^{40}$ T. Gisler, S. F. Schulz, M. Borkovec, H. Sticher, P. Schurtenberger, B. D’Aguanno, and R. Klein, J. Chem. Phys. 101, 9924 (1994).

${ }^{41}$ H. H. von Grünberg, J. Colloid Interface Sci. 219, 339 (1999).

${ }^{42}$ I. Popa, P. Sinha, M. Finessi, P. Maroni, G. Papastavrou, and M. Borkovec, Phys. Rev. Lett. 104, 228301 (2010).

${ }^{43}$ N. Boon and R. van Roij, J. Chem. Phys. 134, 054706 (2011).

${ }^{44}$ T. Palberg, W. Mönch, F. Bitzer, R. Piazza, and T. Bellini, Phys. Rev. Lett. 74, 4555 (1995).

${ }^{45}$ E. Trizac, L. Bocquet, and H. von Grünberg, Langmuir 19, 4027 (2003).

${ }^{46}$ M. Dijkstra and R. van Roij, J. Phys.: Condens. Matter 10, 1219 (1998).

${ }^{47}$ N. Boon, E. Carvajal Gallardo, S. Zheng, E. Eggen, M. Dijkstra, and R. van Roij, J. Phys.: Condens. Matter 22, 104104 (2010). 